\title{
Goat and Bovine Colostrum as a Basis for New Probiotic Functional Foods and Dietary Supplements
}

\author{
Hyrslova $\mathrm{I}^{\star *}$, Krausova $\mathrm{G}^{1}$, Bartova $\mathrm{J}^{2}$, Kolesar $\mathrm{L}^{3}$ and Curda $\mathrm{L}^{4}$ \\ ${ }^{1}$ Dairy Research Institute, Prague, Czech Republic \\ ${ }^{2}$ Clinic of Stomatology VFN, Prague, Czech Republic \\ ${ }^{3}$ Department of Immunogenetics, Institute for Clinical and Experimental Medicine, Prague, Czech Republic \\ ${ }^{4}$ Department of Dairy, Fat and Cosmetics, University of Chemistry and Technology, Prague, Czech Republic
}

\begin{abstract}
Because bovine and goat colostrum are a rich source of essential nutrients for new-borns, their use in functional foods has substantially increased in recent years, but not in combination with probiotic bacteria. Therefore, our aim was to assess the possibility of combining goat or bovine colostrum with probiotics. First, we compared the immunomodulatory effect of both goat and bovine colostrum with that of human milk by the 3-day in vitro stimulation of human peripheral blood mononuclear cells (hPBMCs). Luminex multiplex analysis was used to analyze cytokine production by the hPBMCs. Although goat colostrum had a more similar immunomodulatory effect to human milk than bovine colostrum, we, nevertheless, decided to compare the potential of all three as growth substrates. Using the agar plate method, the growth of bifidobacteria, lactobacilli and enterococci was determined. Growth of Bifidobacterium sp. in goat colostrum is significantly better $(P<0.05)$ than in bovine colostrum or human milk. Our results indicate that goat colostrum has significant potential for the development of new functional foods and dietary supplements with bifidobacteria.
\end{abstract}

Keywords: Colostrum; Cytokines; Probiotics; Bifidobacterium; Lactobacillus

\section{Introduction}

Colostrum is defined as first milk with specific composition produced by mammals immediately after parturition. It contains high levels of bioactive components, including growth factors, immunoglobulins, lactoperoxidase, lactoferrin, lysozyme, immunomodulatory peptides, oligosaccharides and others [1,2]. Bovine colostrum due to content of these antimicrobial components has been tested for the treatment and prevention of various infectious diseases caused by bacteria, viruses and protozoa [3]. Oligosaccharides with prebiotic effect are naturally present in colostrum and can improve the growth of probiotic microorganisms such as lactobacilli and bifidobacteria [4].

During the last twenty years an increase in studies investigating the positive effects of probiotics is observed. Some of the reported beneficial effects of probiotics are associated with immune response modulation and cholesterol lowering ability [5]. Other benefits include prevention and treatment of gastrointestinal diseases and allergies such as colitis, colon cancer and irritable bowel syndrome [6]. The combination of health benefits of probiotics and colostrum can help to supply a market of food supplements and functional food with new products. For this reason, aims of the presented study were to test bovine and goat colostrum as growth substrate for selected probiotic microorganisms and compared the immunomodulatory effect of tested colostrum with human milk on human mononuclear cells.

\section{Methods}

\section{Colostrum}

Bovine colostrum samples were collected from first milking within 2 hours postpartum from cows of Czech pied cattle (Kojcice, Czech Republic). Human milk samples were obtained from voluntary donors from Gynaecology and Obstetrics Clinic of General Faculty Hospital (Prague). Goat colostrum samples were collected from first milking within 2 hours postpartum (Betula Pendula, Czech Republic). After milking of colostrum samples, the samples were immediately frozen and stored at $-20^{\circ} \mathrm{C}$. Colostrum samples were adjusted two different ways. For testing of growth ability of probiotic and potential probiotic microorganisms, milk substrates were pasteurized at $62.5^{\circ} \mathrm{C}$ for $30 \mathrm{~min}$. Samples for immunomodulation were only defatted by centrifugation (6000 g, $15 \mathrm{~min})$ [7].

\section{Immunomodulation}

Samples of human blood were obtained from healthy adult donors from Blood Transfusion Centre of General Faculty Hospital (Prague). Human peripheral blood mononuclear cells (hPBMCs) were isolated from blood by Ficoll-Hypaque gradient (Sigma-Aldrich, Switzerland). Following separation and purification hPBMCs were adjusted at the final concentration of $10^{7}$ cells $\mathrm{mL}^{-1}$. Mononuclear cells $(0.1 \mathrm{ml})$ were stimulated in X-vivo medium (Cambrex, USA) with $0.1 \mathrm{ml}$ of defatted human breast milk or bovine and goat colostrum for 3 days at $37^{\circ} \mathrm{C}$. The total volume was $1 \mathrm{ml}$. Negative control was composed by unstimulated hPBMCs and ex-vivo medium. The microplates with samples were incubated for 3 days at $37^{\circ} \mathrm{C}$. Levels of cytokine produced by stimulation of hPBMCs by different types of colostrum were determined using Fluorokine MAP Human Base Kit A (R\&D Systems, USA) for IFN- $\gamma$, IL-4, IL8, IL-10, IL-12, IL-13 and IL-17 by multiplex analysis using Luminex 200 Analyzer (Luminex Corp., USA).

*Corresponding author: Hyrslova I, Dairy Research Institute, Prague, Czech Republic, Tel: +234-806-7444284; E-mail: hyrslova@milcom-as.cz

Received December 23, 2015; Accepted February 02, 2016; Published February 09,2016

Citation: Hyrslova I, Krausova G, Bartova J, Kolesar L, Curda L (2016) Goat and Bovine Colostrum as a Basis for New Probiotic Functional Foods and Dietary Supplements. J Microb Biochem Technol 8: 056-059. doi: 10.4172/19485948.1000262

Copyright: ( 2016 Hyrslova I, et al. This is an open-access article distributed under the terms of the Creative Commons Attribution License, which permits unrestricted use, distribution, and reproduction in any medium, provided the original author and source are credited. 
Citation: Hyrslova I, Krausova G, Bartova J, Kolesar L, Curda L (2016) Goat and Bovine Colostrum as a Basis for New Probiotic Functional Foods and Dietary Supplements. J Microb Biochem Technol 8: 056-059. doi: 10.4172/1948-5948.1000262

\section{Growth of selected microorganisms in animal colostrum and human breast milk}

Tested strains were obtained from the Culture Collection of Dairy Microorganisms Laktoflora (Czech Republic), from the Czech University of Life Sciences (Czech Republic) and biopsy samples, as shown in Table 1. After overnight cultivation, the tested bacterial cells were isolated from growth medium (Table 1) by centrifugation (6000 g, 7 minutes), washed with sterile saline solution and then finally adjusted in the same buffer at final concentration of $10^{3} \sim 10^{4} \mathrm{CFU} \mathrm{mL}^{-}$ ${ }^{1}$. Before growth testing, colostrum samples were pasteurized at $62.5^{\circ} \mathrm{C}$ for 30 minutes, then cooled at $37^{\circ} \mathrm{C}$ and inoculated with $10 \%(v / v)$ of bacterial suspension. Inoculated samples of colostrum were cultivated in anaerobic jars at $37^{\circ} \mathrm{C}$ for $24 \mathrm{~h}$. The counts of tested strains at 0 and 24 hours were determined using 10 -fold serial dilution and cultivated onto MRS agar (MERCK, Germany) / M17 agar, as shown in Table 1.

\section{Statistical analysis}

The concentration of observed cytokines producing by PBMCs was assessed by software Luminex IS 2.3 (Luminex Corp., USA). All statistical evaluations were evaluated using Kruskal-Wallis test. Differences were considered statistically significant at the level of $\mathrm{P}<0.05$.

\section{Results}

Immunomodulatory effect of human milk, bovine and goat colostrum on human peripheral blood mononuclear cells were compared based on the production of pro-inflammatory and regulatory cytokine. Selected interleukins (IL-1 $\alpha$, IL-4, IL-8, IL-10, IL-17, IL-12 and IL-13) and interferon (INF) $-\gamma$ were determined using multiplex analysis in contrast with other studies where levels of tested cytokines were evaluated by flow cytometry or ELISPOT assay [8,9]. Our results have shown that the production of IL- 8 and IL-12 interleukins was not statistically different $(\mathrm{p}<0.05)$ comparing all three samples. In all the remaining interleukins (ILN- $\gamma$, IL-10, IL-17, IL-1 $\alpha$, IL-4 and IL13) a significant difference between human and bovine colostrum was observed. Immunomodulatory effect of goat colostrum and human milk did not differ significantly (Figure 1).

\begin{tabular}{|c|c|c|c|}
\hline Strain & Species & Origin & Growth condition \\
\hline${ }^{*}$ CCDM 229 & B. animalis ssp. animalis & original culture & \multirow{5}{*}{$\begin{array}{c}\text { MRS broth / agar } 6.2+L \text {-cysteine } \\
\text { hydrochloride; anaerobic, } 37^{\circ} \mathrm{C}, 72 \text { hours }\end{array}$} \\
\hline *CCDM 562 & B. breve & GIT of child & \\
\hline *AVNB3-P1 & B. adolescentis & GIT of child & \\
\hline ***JOV & B. bifidum & infant faeces & \\
\hline$* *$ JKM & B. bifidum & infant faeces & \\
\hline${ }^{*}$ CCDM 150 & L. rhamnosus & curd & \multirow{5}{*}{$\begin{array}{c}\text { MRS broth / agar } 5.7 ; \text { anaerobic } 37^{\circ} \mathrm{C}, 72 \\
\text { hours }\end{array}$} \\
\hline${ }^{*}$ CCDM 66 & L. delbrueckii ssp. bulgaricus & yogurt & \\
\hline${ }^{\star}$ CCDM 151 & L. acidophilus & tabl. Biolacta & \\
\hline${ }^{* *} R L 25$ & L. fermentum & human faeces & \\
\hline *DM1TA6-P & L. casei ssp. paracasei & GIT of child & \\
\hline *CCDM 945 & Enterococcus faecium & original culture & \multirow[t]{2}{*}{ M 17 broth / agar; aerobic $37^{\circ} \mathrm{C}, 72$ hour } \\
\hline${ }^{*}$ CCDM 922 & Enterococcus durans & isolated & \\
\hline
\end{tabular}

"Culture Collection of Dairy Microorganisms Laktoflora, Czech Republic; "*isolate obtained from the biopsy sample of child, "** Czech University of Life Sciences, Prague Czech Republic

Table 1: Tested microorganisms.

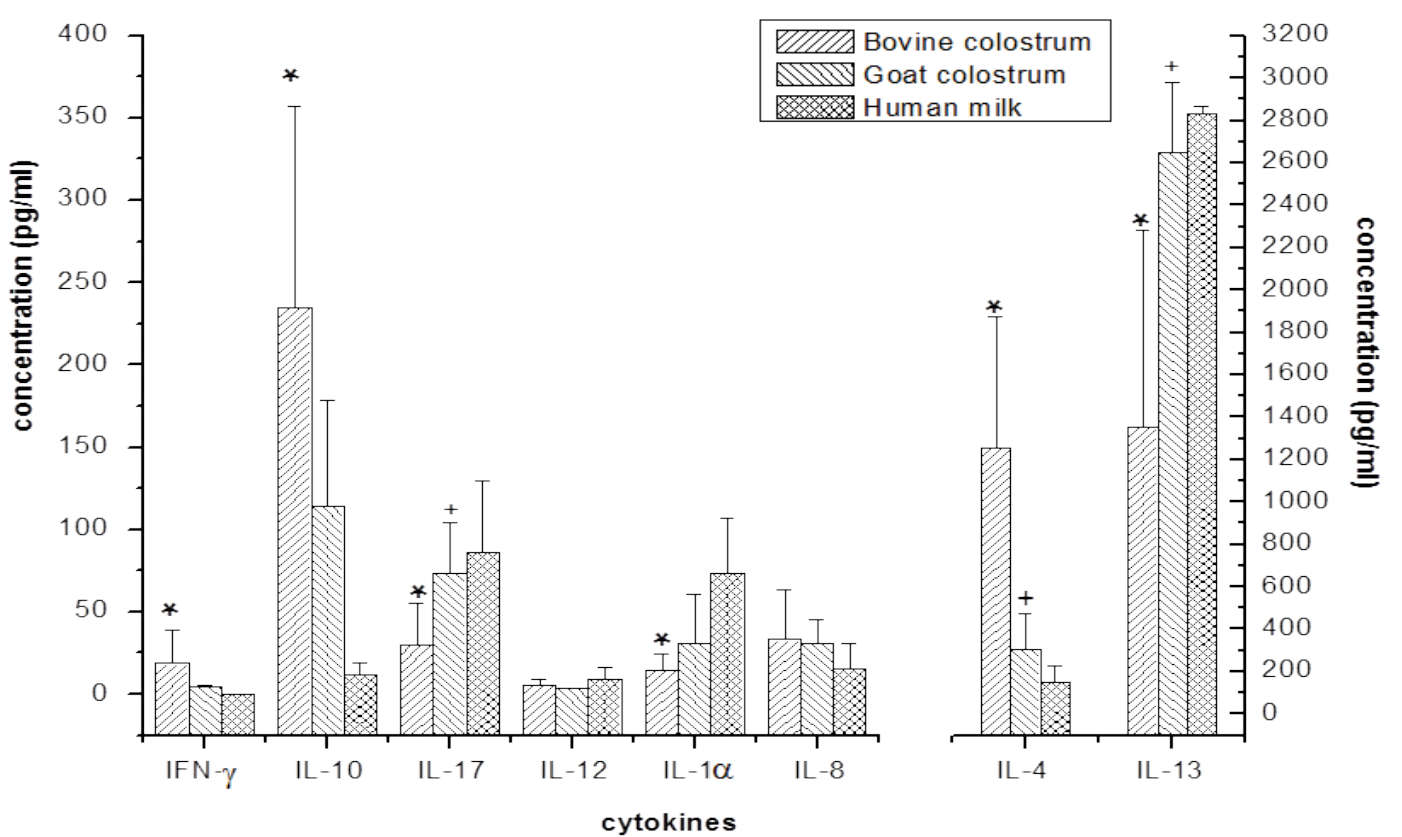

Figure 1: Comparison of IFN-y, IL-10, IL-8, IL-17, IL-4, IL-12 and IL-13 levels (pg mL-1) produced by hPBMCs following 3-days stimulation of human milk, bovine and goat colostrum. 
Although goat colostrum has a more similar immunomodulatory effect to human milk than bovine colostrum, we compared the potential of all three as growth substrates for selected probiotic strains. As shown in Figure 2, we determined statistically significant difference between genera Lactobacillus sp., Bifidobacterium sp. and Enterococcus sp. The growth of Bifidobacterium sp. in goat colostrum was significantly better than in bovine colostrum or human milk (Figure 2). Growth of Enterococcus sp. was significantly lower in human milk than in other two used substrates. Lower counts of tested enterococci could cause their higher sensitivity to antimicrobial substances of human milk.

\section{Discussion}

Colostrum is an important source of protective and imunomodulatory components protecting newborns because their immune system is not fully developed after parturition [10]. Imunomodulatory components of colostrum generally include cytokines, chemokines, imunoglobulines and growth factors [1,11]. During the last years the development of methodological approaches for detection of various biological factors has increased rapidly. Immunoassays (enzymatic immunoassay, RIA) offer generally higher specificity, sensitivity, and reproducibility compared with bioassays [12]. Successfully used methods to detect multiple proteins are also proteomics and antibody-based protein arrays. Antibody-based protein arrays are primarily effective in characterizing the spectra of biologically active components in body fluids and cells $[7,13]$. The advantages of this determination are high numbers of proteins detected on an array and low volume of tested sample required. The protein array is also possible to use in basic and clinical research as well as in clinical practice due to a parallel identification of individual protein biomarkers.

Kverka et al. [12] investigated the cytokine profile in human colostrum and milk by multiplex protein array. They detected the previously described interleukin IL-8, epidermal growth factors (EGF), angiogenin and others, but they also found 32 cytokines which have not been decribed before in these samples. The cytokine spectrum of individual human colostrum and milk was quite variable and the same proteins were not changed consistently over time [12]. High concentrations of cytokines (IL-1 $\beta$, IL- 6 , TNF- $\alpha$, INF- $\gamma$ ), which are included in bovine or goat colostrum, can influence the immune response similarly as those in human colostrum [11,14]. Bovine colostrum also contains other immunomodulatory components (lactoferrin and insulin growth factors (IGF)-1) with the ability to regulate the secretion of cytokines [15]. The immunomodulatory effect of defatted bovine colostrum powder on human mononuclear cells isolated from peripheral blood of healthy donors was also tested in the study of Biswas et al. [2] mononuclear cells were stimulated by two different concentrations of bovine colostrum $(0.1$ and $10.1 \mu \mathrm{g}$ $\mathrm{mL}^{-1}$ ) and after 18-h or 24-h stimulation the production of IL-12 and IFN- $\gamma$ was evaluated. The results have shown that bovine colostrum promoted the production of Th1 cytokine and it can be useful in prevention and treatment of several microbial infections. In present study, bovine colostrum stimulated a high production of IFN- $\gamma$, but also the production of Th2 cytokines IL- 4 and IL-10. Increased production of IL-4 and IL-10 leads to inhibite the production of Th1 cytokines. In other studies of Wan et al. [14] and Yoshiaka et al. [16] tested the effect of oral administration of bovine colostrum on cytokine production in vivo and in vitro in mice. Their results indicate that immunomodulatory effect of bovine colostrum is pleiotropic on the production of cytokine and bovine colostrum can help in keeping the Th1 / Th2 balance.

The growth of tested strains may have been also caused by activity of bioactive components, such as lactoferrin, lysozyme and lactoperoxidase present in tested colostrum and human milk. The antimicrobial effect of bioactive components of milk is influenced by their synergistic manner and it does not depend on the level of their individual contribution [17]. Griffiths et al. [18] tested the ability of human and bovine lactoferrin to influence the growth of B. bifidum, B. infantis, L. acidophilus and enteric bacteria. The results has shown

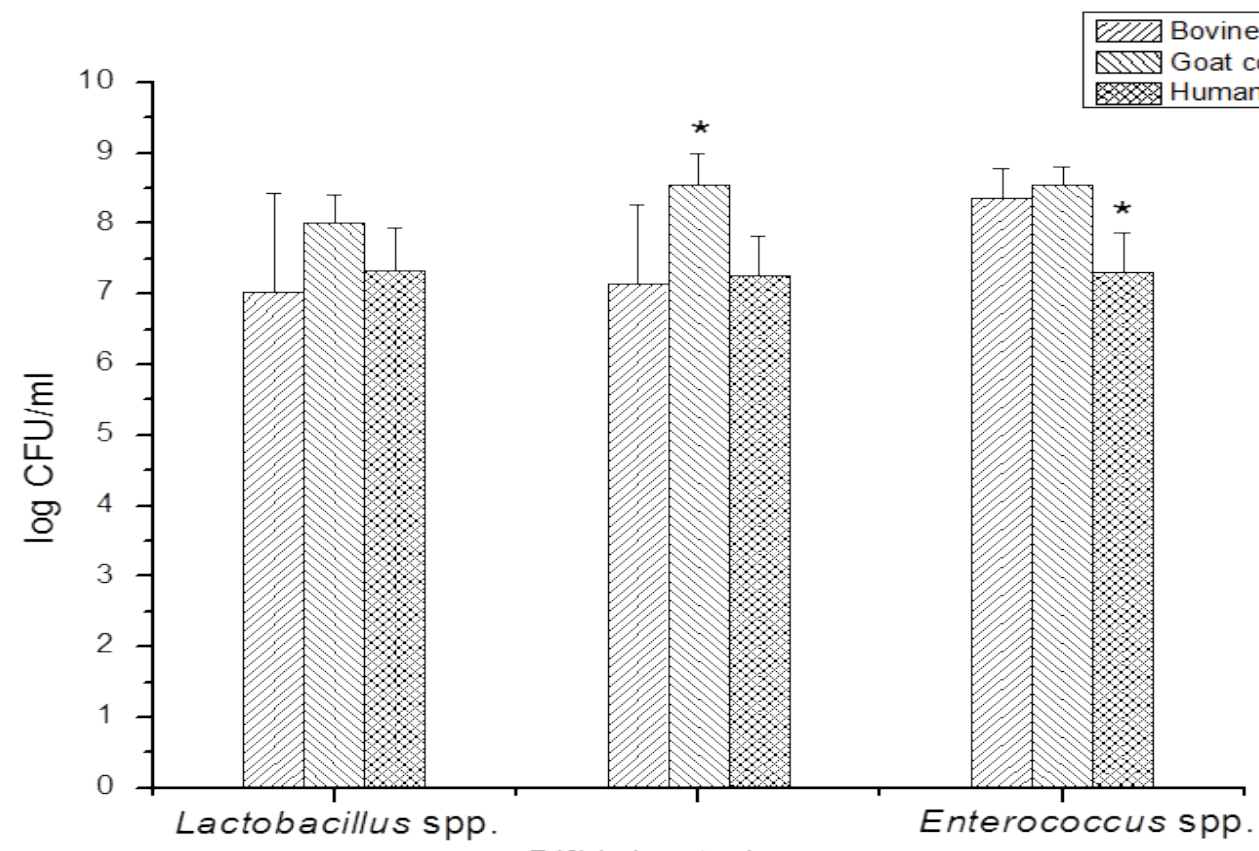

Bifidobacterium spp.

Figure 2: Comparison of growth ability of tested microorganisms in different type of mammalians 
Citation: Hyrslova I, Krausova G, Bartova J, Kolesar L, Curda L (2016) Goat and Bovine Colostrum as a Basis for New Probiotic Functional Foods and Dietary Supplements. J Microb Biochem Technol 8: 056-059. doi: 10.4172/1948-5948.1000262

that lactoferrin in iron-limited forms and probiotics incorporated to biotherapeutic products can inhibit the overgrowth of enteric bacteria and so could help to balance of human gut microflora. In another study, Rada et al. [19] the growth of bifidobacteria in human milk was investigated. The results showed that both tested strains of B. bifidum grew in all human milk samples in contrast to strains of $B$. animalis spp. lactis, in which growth was decreased. B. animalis spp. lactis was the most sensitive to lysozyme in human milk $(15-58 \mu \mathrm{g} / \mathrm{l})$. The concentration of lysozyme in bovine colostrum $(0.14-0.7 \mathrm{mg} / \mathrm{l})$ and its lytic activity is less effective than lysozyme isolated from human milk [20]. Lower lytic activity of bovine lysozyme is caused by a different composition of amino acids than that found in human and albumen lysozyme [21].

In the summary, the results of the present study show that bovine and goat colostrum influences in vitro cytokine secretion of human mononuclear cells. The goat colostrum influenced the secretion of selected cytokines similarly to human milk than bovine colostrum. Nevertheless, its disadvantage is the poor availability and produced quantity. It is necessary to remark that this study has tested only a limited range of cytokines and that both colostrum may influence other cytokines and chemokines which play a role in immunomodulation. Further determination into the mechanism of cytokine production from human mononuclear cells and specific cell populations stimulated by mammalians colostrum or combination of colostrum and probiotics is warranted.

\section{Acknowledgement:}

This study was supported by Ministry of Agriculture of the Czech Republic, Decision No RO1415 and project No QJ1210376.

\section{References}

1. Uruakpa FO, Ismond MA, Akobundu M (2002) "Colostrum and its benefits: a review," Nutr Res 22: 755-767.

2. Biswas $P$, Vecchi $A$, Mantegani $P$, Mantelli $B$, Fortis $C$ (2007) Immunomodulatory effects of bovine colostrum in human peripheral blood mononuclear cells. New Microbiol 30: 447-454.

3. Stelwagen K, Carpenter E, Haigh B, Hodgkinson A, Wheeler TT (2009) Immune components of bovine colostrum and milk. J Anim Sci 87: 3-9.

4. Playford J (2000) Peptide therapy and the gastroenterologist: colostrum and milk-derived growth factors. Clinical Nutrition 20: 101-106.
5. Nagpal R, Kumar A, Kumar M, Behare PV, Jain S, et al. (2012) Probiotics, their health benefits and applications for developing healthier foods: a review. FEMS Microbiol Lett 334: 1-15.

6. Saad N, Delattre C, Urdaci M (2013) An overview of the last advances in probiotic and prebiotic field. LWT-Food Sci Technol 50: 1-16.

7. Žižka J, Hrdý J (2007) Effect of breast milk of healthy and allergic mothers on in vitro stimulation of cord blood lymphocytes. Pediatr Allergy Immunol 18: 486-489.

8. Mulder M, Connellan P, Oliver J (2008) Bovine lactoferrin supplementation support immune and antioxidant status in healthy human males. Nutr Res 28 : 583-589.

9. He F, Tuomola E, Arvilommi H, Salminen S (2001) Modulation of human humoral immune response through orally administered bovine colostrum FEMS Immunol Med Microbiol 31: 93-96.

10. Shing M, Peake M (2009) Bovine colostrum modulates cytokine production in human peripheral blood mononuclear cells stimulated with lipopolysaccharide and phytohemagglutinin. J Interferon Cytokine Res 29: 34-37.

11. Hagiwara K, Kataoka S, Yamanaka H, Kirisawa R, Iwai H (2000) Detection of cytokines in bovine colostrum. Vet Immunol Immunopathol 76: 183-190.

12. Kverka M, Burianova J (2007) Cytokine profiling in human colostrum and milk by protein array. Clinical chemistry 53: 955-996.

13. Joos TO, Stoll D, Templin MF (2002) Miniaturized multiplexed immunoassays Curr Opin Chem Biol 6: 76-80.

14. Wan Z (2010) Effect of orally administered bovine colostrum on cytokine production in vivo and in vitro in immunosuppressed mice. Int dairy J 20: 522-527.

15. Ginjala V, Pakkanen R (1998) Determination of transforming growth factorbeta 1 (TGF-beta 1) and insulin-like growth factor (IGF-1) in bovine colostrum samples. J Immunoassay 19: 195-207.

16. Yoshioka $Y(2005)$ Oral administration of bovine colostrum stimulates intestinal intraepithelial lymphocytes to polarize Th1-type in mice. Int J Immunopharmacol 5: 581-590.

17. Benkerroum N (2008) Antimicrobial activity of lysozyme with special relevance to milk. Afr J Biotechnol 7: 4856-4867.

18. Griffiths EA, Duffy LC, Schanbacher FL, Dryja D, Leavens A, et al. (2003) In vitro growth responses of bifidobacteria and enteropathogens to bovine and human lactoferrin. Dig Dis Sci 48: 1324-1332.

19. Rada V (2010) Susceptibility of bifidobacteria to lysozyme as a possible selection criterion for probiotic bifidobacterial strains Biotechnol 7: 451-455.

20. Solomons NW (2002) Modulation of the immune system and the response against pathogens with bovine colostrum concentrates. Eur $\mathrm{J}$ Clin Nutr 56 Suppl 3: S24-S28.

21. Pakkanen R, Aalto J (2002) Growth factors and antimicrobial factor of bovine colostrum. Int Dairy J 7: 285-297. 